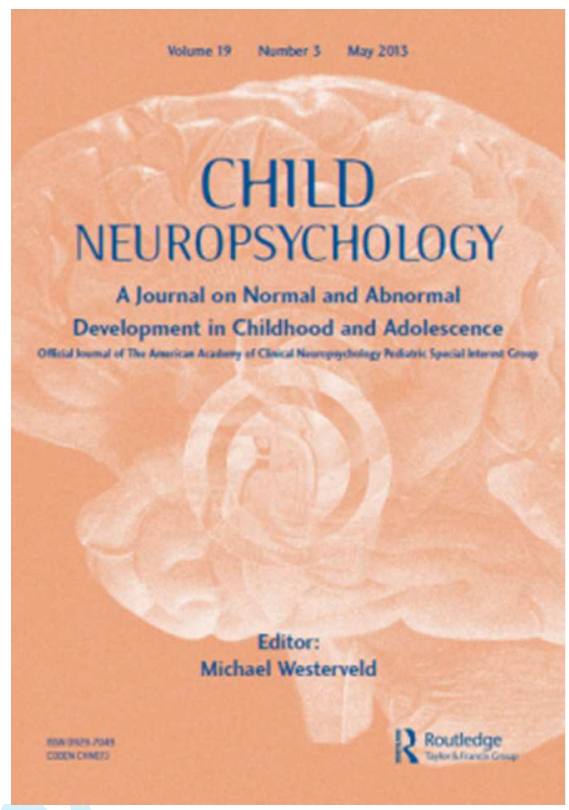

\title{
Executive abilities in children with congenital visual impairment in mid-childhood
}

\begin{tabular}{|r|l|}
\hline Journal: & Child Neuropsychology \\
\hline Manuscript ID & CNY-OA 16-50.R1 \\
\hline Danuscript Type: & Original Article \\
\hline Complete List of Authors: & $\begin{array}{l}\text { Bathelt, Joe; Medical Research Council, Cognition \& Brain Sciences Unit } \\
\text { De Haan, Michelle; UCL Institute of Child Health, Division of Developmental } \\
\text { Neurosciences } \\
\text { Salt, Alison; Great Ormond Street Hospital For Children NHS Trust, } \\
\text { Developmental Vision Clinic; Moorfields Eye Hospital NHS Foundation Trust } \\
\text { Dale, Naomi; Great Ormond Street Hospital For Children NHS Trust, } \\
\text { Developmental Vision Clinic; University College London, Institute of Child } \\
\text { Health }\end{array}$ \\
\hline Keywords: & $\begin{array}{l}\text { visual impairment, mid-childhood, executive function, attention, working } \\
\text { memory, cognitive development }\end{array}$ \\
\hline
\end{tabular}

\section{SCHOLARONE $^{\text {Im }}$}

Manuscripts 


\title{
Executive abilities in children with congenital visual impairment in mid-childhood
}

\author{
Joe Bathelt ${ }^{1}$, Michelle de Haan², Alison Salt ${ }^{3}$, Naomi Jane Dale ${ }^{34}$ \\ ${ }^{1}$ Cognition \& Brain Sciences Unit, Medical Research Council \\ ${ }^{2}$ Cognitive Neuroscience \& Neuropsychiatry Section, Division of Developmental \\ Neurosciences, UCL Institute of Child Health \\ ${ }^{3}$ Developmental Vision Service, Great Ormond Street for Children NHS Foundation Trust \\ ${ }^{4}$ Clinical Neurosciences Section, Division of Developmental Neurosciences, UCL Institute \\ of Child Health
}


1

2

3

4

5

6

7

8

9

10

11

12

13

14

15

16

17

18

19

20

21

22

23

24

25

26

27

28

29

30

31

32

33

34

35

36

37

38

39

40

41

42

43

44

45

46

47

48

49

50

51

52

53

54

55

56

57

58

59

60
24 Keywords: visual impairment, mid-childhood, executive function, attention, 25 working memory, cognitive development 
Executive functions (EF) are highly important for educational attainment and academic success in childhood and adolescence (de Haan, 2014; McDermott, Westerlund, Zeanah, \& Fox, 2012; Stevens, Lauinger, \& Neville, 2009). EF is used as an umbrella term for a set of inter-related cognitive abilities, including goal planning, control of attention, working memory, inhibition, and cognitive flexibility (Anderson, 2002; Diamond, 2013). Current theoretical models about the early development of executive function are largely based on observations of visual behaviors though the importance of early vision for early and later EF development is unknown (Colombo, 2001; Johnson \& de Haan, 2011; Richards, Reynolds, \& Courage, 2010). A link between vision and executive function is possibly suggested by the close connection between visual processing streams with prefrontal regions and the fronto-parietal attention network Kravitz, Saleem, Baker, \& Mishkin, 2011; Ptak, 2011) and also the close relationship between visuo-spatial working memory, spatial abilities, and EF (Mikaye et al 2001). However, it is currently not clear if early or later visual behaviors are necessary for the development of executive abilities and the integration of executive function networks in the brain or if experiences in other non-visual modalities (auditory, haptic) are sufficient for the development of executive functions in the absence of vision. Consequently, studying the development of children with congenital visual impairment (VI) may shed light on the relationship between EF and vision, and visual experience and potential vulnerabilities or compensatory factors in the development of EF abilities in this clinical population. In addition, this is of high clinical and educational importance as children with VI may have to rely more on their ability to plan, organize, and hold information in working memory when visual cues are inaccessible.

Congenital visual impairment is associated with differences of large-scale structural and functional brain network organization (Liu,Yu, Liang, Li, Tian, Zhou, Qin, Li, \& Jiang 2007, Shu, Li, Li, Yu, \& Jiang 2009, Noppeney, 2007), which may affect the distributed networks involved in executive function (Cavezian, Vilayphonh, Vasseur, Caputo, Laloum, \& Chokron, 2013). Of relevance, evidence from an observational study indicated differences in potential precursors of executive behaviors, specifically attention shifting, in preschoolers with VI compared to matched typically-sighted (TS) peers (Tadic, Pring, \& Dale, 2009). The authors reported reductions in the frequency at which preschoolers with severe to profound VI responded to adult attempts to elicit or maintain their attention and in particular to shift their attention from one object to another through auditory, haptic or visual cues, with greatest difficulty in those with profound VI (light perception at best). Interestingly, individual weaker response to attention shifting was significantly related to more problems in everyday behaviors requiring EF on the Behavior Rating of Executive Function (BRIEF) questionnaire, in particular Shifting, when the same children were seen at school age (Tadic, 2009). Neurodevelopmental differences that are potentially related to EF have also been reported in mid-childhood to adolescence in other samples of children with VI. A comprehensive survey in a sample of 264 children (aged 4-17 years) attending specialist clinics found a substantially higher prevalence of Attention Deficit Hyperactivity Disorder (ADHD) diagnoses in children with VI (22.9\% compared to $14.3 \%$ in the TS population in the same geographical area) (Decarlo, Bowman, Monroe, Kline, McGwin, \& Owsley, 2014); EF has been shown to be a significant component in ADHD in sighted samples (Willcutt, Doyle, Nigg, Faraone, \& Pennington, 2005). Further, a recent study by Greenaway and colleagues indicated higher parental ratings of behavioral executive functioning deficits, compared with normative population expectations, in a small sample of high-functioning adolescents with con- 
genital VI and age-appropriate verbal IQ (Greenaway, Pring, Schepers, Isaacs, \& Dale, 2016).

77 Whilst these preliminary small scale studies suggest that behaviors related to EF may be negatively affected in children with VI, it is not clear how VI might impact on EF and whether specific aspects of EF are more vulnerable than others. Certain executive abilities might be more dependent on visual information during development, whereas other abilities may develop typically or are more amenable to compensatory mechanisms even when visual information is largely inaccessible or very degraded. The auditory and haptic modalities, and the mechanism of language, have been proposed to modulate developmental processes in the absence of vision (Warren, 1994, Perez-Pereira and ContiRamsden, 1999).

In this study, we therefore set out to investigate the development of EF in the context of congenital VI during mid-childhood. This period has been argued as important for EF development as rapid advances in executive ability have been observed in this age period (Xu et al., 2013). Further, executive abilities are believed to be more differentiated in mid-childhood compared to preschool years (Anderson, 2002; Diamond, 2013) allowing for a more fine-grained assessment of the possible impact of VI on EF development. To investigate this potential relationship, this study focused on children with congenital visual disorders. The subpopulation of interest was those with disorders affecting the anterior or peripheral part of the visual system with no known involvement of central brain structures according to the visual disorder diagnosis (i.e. 'potentially simple' congenital disorders of the peripheral visual system - CDPVS, Sonksen \& Dale, 2002). In children with additional brain defects, as is common in cerebral VI (Rahi, Cable, BCVISG, 2003), the likelihood of comorbid learning difficulties is greatly increased. This would pose a significant confound as any differences in cognitive performance may be potentially linked to the learning disability rather than to the impact of vision reduction per se (Sonksen and Dale 2002). To further minimize the possibility of additional learning difficulties which can commonly occur in children with congenital VI (Alimovic, 2013), a sample of higher functioning children with VI and normal range verbal intelligence were selected for the study. Standard auditory and verbal assessments of EF were employed including assessments of working memory, auditory attention, and verbal fluency to cover a range of executive tasks that did not require vision for performance. In addition, parents filled in a standard questionnaire on everyday behaviors associated with EF. To test further the relationship between vision level and $\mathrm{EF}$, children with differing degrees of VI (from profound/ severe - P/SVI to moderate/mild - MVI) were included in the VI sample; this permitted comparison of a broad spectrum of children with congenital visual disorders with sighted controls and also comparison of different degrees of vision and vision reduction (P/SVI versus MVI versus TS). The study design was therefore selected to permit novel insight into the potential impact of congenital vision reduction on EF in middle childhood, including comparison of those who remained profoundly or severely visually impaired with those who had continued to develop significant functional visual acuity by middle childhood. Previous research of younger children had suggested that those with the most profound VI (especially light perception at best) had the greatest developmental impact with significant delays in cognition, language and social develop- 
ment (Dale and Sonksen 2002, Dale et al 2013), leading us to predict that children with no or very low vision would also show negative impact in EF abilities.

Assuming visual input is necessary for the typical development of EF, it is hypothesized that children with VI have lower standard scores on auditory tests of EF compared with typically sighted matched controls, and that the scores would be even lower in children with the greatest severity of vision reduction (P/SVI) compared with those with moderate vision reduction (MVI) or typically sighted. Standard neuropsychological assessment measures with good construct validity that make no demand on vision were selected for this study. In the absence of well validated tactile or haptic assessments, these measures were either auditory or verbal. The only available auditory tasks that were suitable for children with VI and were all arguably tapping into EF were those of working memory, auditory attention and verbal fluency (Delis, Lansing, Houston, Wetter, Han, Jacobson, 2001; Jurado \& Rosselli, 2007; Manly, Nimmo-Smith, Turner, Watson, \& Robertson, 2001). A parent rated standard questionnaire measure was also included to assess performance and any difficulties with everyday behaviours associated with executive abilities.

\section{Methods}

\section{Participants}

This project was approved by the NHS Paediatric Research Ethics Committee (Ref: 12/LO/0939). Written consent was obtained from all parents/guardians according to the Declaration of Helsinki.

A prospective cross-sectional study was undertaken with eighteen children with VI aged between 8 and 13 years. Congenital disorders of the peripheral visual system with severe VI are rare with an estimated prevalence of less than 2-3 per 10,000 children (UK) raising challenges for recruitment and sampling (Rahi, Cable, BCVISG, 2003). Children were therefore recruited through national specialist clinics at Great Ormond Street Hospital for Children NHS Foundation Trust and Moorfields Eye Hospital NHS Foundation Trust. The investigations reported here were part of a larger study to investigate neural, cognitive and behavioral correlates in this sample. Inclusion criteria: 1) 'potentially simple' congenital disorders of the peripheral visual system (CDPVS, see Sonksen and Dale 2002), i.e. any visual disorder affecting the globe of the eye, the retina, or the anterior optic nerve up to the optic chiasm and no other known central nervous system involvement or brain insult in the pediatric diagnosis; originally diagnosed by paediatric ophthalmology, 2) English as a first language or relatively fluent level of English to participate in assessments, 3 ) children within the normal range for verbal reasoning (>VIQ 79). Identification of children was initially through clinical databases and also self- re- 
IQ slightly below the inclusion criterion. This participant did not act as an outlier on other assessments and was therefore retained in the analysis.

Exclusion criteria: 1) hearing impairment and severe motor impairment, 2) retinopathy of prematurity, 3) pediatric diagnoses of comorbid neurological disorders or indication of other brain involvement or endocrine abnormalities, e.g. hypopituitarism (Garcia-Filion \& Borchert, 2013). Control sample: Eighteen children with normal or corrected-to-normal vision were recruited through local advertisement to match according to age. Children in the control group had to attend mainstream school at age-appropriate level and have no known neurological or psychiatric conditions and have English as a first language.

Sample characteristics are summarized in Table 1. The experimenter (J.B.) was trained by a neurodisability pediatrician specialized in VI (A.S.) to undertake the visual acuity assessments using the Sonksen logMAR test of Visual Acuity (Sonksen, Wade, Proffitt, Heavens, \& Salt, 2008). For children, who were not able to see the largest items on the Sonksen logMAR test, the Near Detection Scale was used to assess their basic level of detection vision (Sonksen, Petrie, \& Drew, 1991).

Severe/Profound VI (S/PVI) is defined as limited form vision with logMAR above 0.8 (Snellen worse than 6/36) to no or light perception only (Near Detection Scale). Mild/moderate VI (MVI) is defined as reduced visual acuity with logMAR between 0.6 182 and 0.8 (Snellen 6/24-6/36).

Table 1 here

185 Table 2 here

\section{Procedure and testing environment}

187 Participants were tested by an experimenter trained in the assessment of children with 188 VI (J.B.) under the supervision of a clinical psychologist specialized in VI (N.D.). Assess189 ments were carried out in a quiet testing room in the university hospital center. Children 190 were given frequent breaks between assessments to maintain optimal performance and 191 promote participant wellbeing.

\section{Verbal comprehension}

193 In order to exclude the possibility that any difference in EF may be due to underlying 194 differences in intellectual ability, a standard test of verbal comprehension was adminis195 tered. Verbal comprehension was assessed using verbal subtests of the Wechsler Intelli196 gence Scale for Children 4th edition (WISC-IV) (Wechsler, 2004). Verbal subtests of pre197 vious and current editions of the WISC have also been used with children with VI 198 (Greenaway et al 2016, Dekker, 1993; Tillman, 1973; Tillman \& Bashaw, 1968; Witkin et 199 al., 1968). 
The administered subtests included all items of the Verbal Comprehension composite score (Vocabulary, Similarities, Comprehension). Two items were altered that required direct visual experience: The WISC-IV first practice item on the Similarities subtest which includes colour was not administered. The Comprehension question that asks about a situation in which 'you see thick smoke' was changed to 'you smell thick smoke'. These alterations were used for the whole sample, including the TS control group. All other items were administered verbatim according to the WISC-IV administration manual (Wechsler, 2004). significant differences between the groups (S/PVI: mean=100.78, SE $=8.94$, Range $=75$ 148; MVI: mean=103.25, $\mathrm{SE}=3$, Range=93-116; $\mathrm{TS}$ : mean=113.17, $\mathrm{SE}=3.87$, Range=83-

\section{EF tasks}

\section{Working memory}

Tasks comprising the Working Memory (WM) composite of the Wechsler Intelligence

218 Scale for Children 4th edition (Wechsler, 2004) were administered to determine work-

219 ing memory performance. The WM composite was calculated from the Digit Span and

220 Letter-Number Sequence scale scores.

\section{Sustained \& Divided Auditory Attention}

Auditory attention was assessed through tests from the Test of Everyday Attention for Children (TEA-Ch) (Manly, Nimmo-Smith, Turner, Watson, \& Robertson, 2001). In the Score! subtest, children had to count infrequently presented sounds in several trials over a 6 min period. Because of long pauses between tones and simple task demands, children have to actively sustain their attention to perform the task (Anderson, 2002). The Score Dual Task condition requires children to count the number of scoring sounds while listening out for an animal name in a simultaneously presented news broadcast (Manly,

230 Nimmo-Smith, Turner, Watson, \& Robertson, 2001).

\section{Verbal Fluency}

232 The Verbal Fluency task of the Delis-Kaplan Executive Function System (D-KEFS) (Delis, 233 Lansing, Houston, Wetter, Han, Jacobson, 2001d) consists of three conditions. In the Let234 ter Fluency (LF) condition, the participant has to name as many words as possible that 235 start with a given letter within 60s. In the Category Fluency (CF) task, the participant has 
to name words within 60 s that belong to a semantic category. In the third condition, Category Switching (CS), participants have to switch between words that belong to different semantic categories. All tests were administered according to the test manual. The DKEFS Verbal Fluency subtest typically requires the assessor to talk through the rules as well as present them visually in print. As the participants were unable to access the print, the assessors ensured that the participants had understood the rules by talking through these carefully and clearly, providing repetition if required. Two children did not complete the task. Seventeen children in the VI (7 male, 8.2713.32y, WISC Verbal Comprehension: 75-148) and 17 children in the control group (10 male, 8.56-12.92y, WISC Verbal Comprehension: 83-144) completed the Verbal Fluency tasks.

\section{Everyday Executive Skills}

The Behavioral Rating Inventory of Executive Function (BRIEF) is an 86 item questionnaire suitable for children aged 5 to 18 years (Gioia, Isquith, \& Kenworthy, 2000). The questionnaire rates executive skills in domains of Inhibition, Shifting, Emotional Control, Initiation, Working Memory, Planning/Organizing, Organization of Materials and Monitoring. Only one of the items used to create these scores makes a reference to visual behavior (Item 31: "Has poor handwriting"), but does still apply to the majority of children in this study with mild to moderate VI. Two additional items may be indirectly related to vision, e.g. Item 67: "Cannot find things in room or school desk" and Item 68: "Leaves messes wherever he/she goes", but also reflect executive contributions. These tasks may be harder for children with visual impairment, but do not necessarily depend on vision. For this reason, parents were given the full questionnaire without any modifications.

Inconsistency scores were below the 98th percentile and were therefore in the acceptable range according to the questionnaire manual. There were two cases of highly elevated Negativity scores in the VI group (above the 98\%ile). High negativity scores may indicate an excessively negative attitude of the rater, but may also suggest extreme executive dysfunction (Gioia, Isquith, \& Kenworthy, 2000). Separate analysis showed no effect of the inclusion or exclusion of these cases for the group results. Therefore, the presented results include cases with high negativity ratings.

\section{Statistical analysis}

Statistical analysis was based on analysis of variance (ANOVA) models. Mauchly's test was used to assess violations of the sphericity assumption (Mauchly, 1940). In the case of violated sphericity assumptions, the Greenhouse-Geisser correction was applied (Greenhouse \& Geisser, 1959). All statistical tests were performed in R v2.15.3 (The R Development Core Team, 2008). Follow-up contrasts were based on Student's t-tests . Welch correction was applied to account for difference in variance between the groups 
274 (Welch, 1947). Visualization were based on ggplot2 algorithms (Wickham, 2009). A sig-

275 nificance level of $\mathrm{p}<0.05$ was used for all statistical analyses. Values between 0.05 and

$276 \quad 0.1$ are discussed as trend-level effects.

277

9

10

11

12

13

14

15

16

17

18

19

20

21

22

23

24

25

26

27

28

29

30

31

32

33

34

35

36

37

38

39

40

41

42

43

44

45

46

47

48

49

50

51

52

53

54

55

56

57

58

59

60 
Figure 1 about here

Table 3 about here

\section{Working Memory}

284 Statistical analysis did not indicate significant differences in the Working Memory composite score between the vision groups (S/PVI, MVI, TS) $(\mathrm{F}(2,31)=0.079, \mathrm{p}=0.971$, see Table 3 for descriptive statistics). There was also no significant effect of vision group on the Digit Span $(\mathrm{F}(2,32)=0.824, \mathrm{p}=0.448)$ or Letter-Number Sequence score $288 \quad(\mathrm{~F}(2,32)=1.033, \mathrm{p}=0.368)$.

\section{Sustained \& Divided Auditory Attention}

Statistical analysis did not indicate significant differences in the Working Memory composite scores between vision groups $(F(2,32)=0.515, p=0.602$, see Figure $1 \mathrm{a}$ and Table 3 for descriptive statistics). There was also no significant effect of vision group in the divided attention condition $(\mathrm{F}(2,32)=1.599, \mathrm{p}=0.218)$. A high proportion of participants in both groups reached scores in the superior to highly superior range compared to the normative sample of the test (see Figure 1b). However, there was also considera-

\section{Verbal Fluency}

301 Statistical analysis did not indicate significant differences in the Letter Fluency scores 302 between vision groups $(\mathrm{F}(2,32)=0.711, \mathrm{p}=0.499$, see Figure $1 \mathrm{a}$ and Table 3 for descrip303 tive statistics). Category Fluency condition: There was no significant effect of vision 304 group on category fluency scores $(\mathrm{F}(2,30)=0.737, \mathrm{p}=0.487)$. There was also no signifi305 cant effect of vision group on the number of responses in the switching condition $306 \quad(\mathrm{~F}(2,30)=0.128, \mathrm{p}=0.88)$ or switching accuracy $(\mathrm{F}(2,30)=0.314, \mathrm{p}=0.733)$.

\section{Everyday executive skills}

309 Half of the children with VI reached threshold for clinical concern regarding execu310 tive deficits on the BRIEF (9 children (50\%, 4 MVI, 5 S/PVI) over GEC cut-off at 65 , 
$311>93 \%$ ile). Statistical comparison indicated a main effect of vision group $(\mathrm{F}(2,27)=4.444$,

$312 \mathrm{p}=0.022$ ). Follow-up contrasts indicated significantly higher scores in severe/profound

313 group compared to controls $(\mathrm{t}(10.58)=2.806, \mathrm{p}=0.018)$. Other contrasts did not reach

314 significance level.

315 Eight children with VI scored above the cut-off on the Behavioral Regulation Index 316 (BRI) (45\%, 4 MVI, 4 SVI, cut-off at 65, >93\%ile). Scores on the BRI also showed a signif317 icant effect of vision group $(\mathrm{F}(2,27)=6.248, \mathrm{p}=0.006)$. Post-hoc contrasts revealed a sig318 nificantly higher score in the severe/profound compared to the control group $319(\mathrm{t}(7.827)=2.339, \mathrm{p}=0.048)$ and a trend-level difference between the mild/moderate and 320 control group with higher scores in the MVI group $(t(8.851)=-2.171, p=0.058)$. There 321 was no significant difference between the two VI groups.

Seven children with VI reached scores above the cut-off on the Metacognitive Index (MI) (38\%, 3 MVI, 4 S/PVI, cut-off at 65, >93\%ile). Statistical analysis also indicated a 324 significant difference between vision groups on the $\mathrm{MI}(\mathrm{F}(2,27)=8.020, \mathrm{p}=0.001)$. Fol325 low-up contrasts indicated significantly higher scores in the S/PVI compared to controls $326(\mathrm{t}(3.82)=8.127, \mathrm{p}=0.005))$ as well as a trend-level difference between the MVI and con327 trol group with higher scores in the MVI group $(t(8.134)=-2.01, p=0.079)$. The differ328 ence between the VI groups (MVI vs S/PVI) was not statistically significant $(\mathrm{t}(13.683)=-$ 329 0.405, $\mathrm{p}=0.692$ ).

330

331 


\section{Discussion}

334

The relationship between congenital VI and EF abilities in middle childhood has not been studied systematically before and the available theories and evidence suggest that lack of vision and deprivation of visual information from the environment might impact adversely on the developmental or behavioral aspects of EF. Nevertheless, alternative sensory functions of audition and touch might provide compensatory avenues for developing EF abilities. To investigate this further, this study focused on the performance of EF abilities in a sample of 18 children with congenital VI in middle childhood, compared with typically-sighted and typically developing controls (TS). The precautionary methodological approach to reduce potential confounding influences of comorbid learning disability (which is high in children with congenital visual disorders) or an inability to perform the EF task because of lack of vision to see the materials included adopting 1) children with VI in the 'simple' CDPVS subpopulation, 2) only higher functioning range of verbal intelligence and 3) no tasks requiring vision. Contrary to arguments leading to us hypothesizing that EF abilities might be adversely constrained in children with VI and particularly in the most severe VI (light perception or low levels of 'form' vision), we found no significant differences in standard scores of EF tasks of working memory, sustained and divided attention, phonemic, semantic, and switching verbal fluency between the VI and the age-matched TS groups. Moreover, the mean standard scores of the VI group were on average in line with age-appropriate population norms.

A number of theoretical positions could explain this 'typical' performance in EF neuropsychological tasks in children with VI. Firstly, infancy and later experience in auditory and haptic sensory modalities, including possibly the mediating role of language and non-verbal physical and object experiences, has assisted the development of metacognitive thought processes and mental abstraction involved in executive skills. In terms of the possible origin of EF abilities in childhood, present theoretical models of possible precursors in infant behavior are largely based on the observation of visually-mediated behaviors, like saccades (Colombo, 2001; Richards, Reynolds, \& Courage, 2010). For instance, the ability to shift visual fixation from an intrinsically attractive visual stimulus to a less intrinsically attractive, but task-relevant visual stimulus is seen as a precursor of top-down executive control in longitudinal studies (Nakagawa et al., 2013; Papageorgiou et al., 2014). To the authors' knowledge, there are currently no theories of infancy EF development based on other auditory or haptic modalities, potentially due to methodological difficulties in assessing these functions in infants though auditory oddball paradigms may be revealing in the future (Gomes et al., 2000). Investigations of auditory or haptic (tactile) aspects of EF precursors in infants with congenital VI will need to be pursued, though the methodological challenges cannot be understated. Our finding in relation to auditory EF function raises the possibility that alternative non-visual modalities 
may provide a compensatory route to the development of EF.

Secondly, there may be modality-specific executive skills that are tied to the availability of sensory information and, in this sense, verbal and auditory EF skills would be expected to develop smoothly to a relatively preserved level in children with VI. Others have also argued for the important role of verbal ability in EF function in childhood (Henry, Messer and Nash 2012). A related model to this is that if children with VI are restricted to modality-specific EF skills, then they might be expected to have much greater difficulty in areas of EF that are associated with vision, such as design fluency and spatial working memory. This dimension was not explored in this paper but is worthy of further investigation to see if effects are amodal or modality-specific.

A third possible model is that EF abilities are a unitary construct in middle childhood. If EF is unitary in middle childhood and our sample of children with VI scored in the age-appropriate range comparable to the TS sample, then one might deduce that EF is amodal in middle childhood and can be executed through visual or auditory/verbal means. Xu et al (2013) demonstrated that from 5-7 and 8-11 years children's performance on different executive function tasks was found to be explained best by a singlefactor model rather than the three factor model of working memory, inhibition, and shifting commonly described for adults (Miyake, Friedman, Emerson, Witzki, Howerter, $\&$ Wager, 2000). The similar performance on the different auditory neuropsychological tests in both the VI and control groups could reflect the early unitary nature of executive function. This might explain the finding that the children with VI did relatively well on all aspects of EF assessment tasks tested in this study. Executive abilities might diversify into discrete EF abilities in adolescence with differences between VI and TS participants emerging at this later developmental stage. The preliminary results presented by Greenaway et al. 2016 suggest that this may be the case. However, despite apparent similar abilities on the group level, some individual children with VI displayed highly uneven neuropsychological profiles with extreme weakness in certain tasks (see too Greenaway et al 2016 in higher functioning adolescents with VI). For reasons not yet understood, there was extreme variation between and within some of the individual children with scores ranging from extremely low to superior level. A more detailed investigation of the potentially multiple factors contributing to these individual differences (Sonksen and Dale 2002) will only be possible through assessment of larger samples in future studies.

In contrast to test performance, results of the behavioral ratings (BRIEF, parent rating) showed significant differences between the VI and TS groups and indicated around half of the children with VI reached clinical threshold for EF difficulties (>93\%ile). According to expectations for typically-sighted children, these scores would indicate significant difficulties in the domains of behavioral regulation and metacognition. These findings replicate the results of an independent sample of 6-12-year-old children with severe to profound VI and typical intelligence (Tadic, Pring, 2009) and re-

411 sults based teacher reports in a wider sample of children with VI (Heyl and Hintermair 412 2015). Further, the current study provides evidence that behavioral executive function- 
ing is also affected in some children with mild to moderate VI.

414 It has been argued that standardized neuropsychological tests of EF place reduced demand on executive skills by providing an adult directed environment with clear instructions, training items, and a problem-solving scaffold. These aids are rarely available in everyday dynamic situations requiring EF abilities such as taking initiative, generating new ideas, making plans, achieving goals and self-organization of materials

\section{Limitations} (Isquith et al., 2013). The discrepancies found in this study between the assessment scores and the parent ratings might therefore indicate that 'core' cognitive skills in standard EF tasks are similar in both the VI and the control group, but that everyday demands on dynamic performance requiring executive skills are much higher for children with VI, e.g. lack of access to visual information from the environment may increase the cognitive load of a task (Bertone et al. 2007) and reduce the environmental supports for basic mobility and orientation required in executing any physical or goalfocussed activity (Warren 1994). This argument is further reinforced by the finding that more severe levels of VI (S/PVI vs. MVI group comparisons) were significantly associated with more everyday behavioral executive difficulties. Further, children with S/PVI who are likely to receive more assistance may have less opportunity to practice relevant behavior leading to less proficiency at performance level, despite intact 'core' skills.

Alternatively, the current findings could be explained by both higher vulnerabilities in the VI group in some EF skills, such as taking initiative or achieving goals, in addition to higher performance demands particularly in the children with the most severe VI. Moreover, further evidence of a highly similar discrepancy between test performance on similar neuropsychological EF tasks and the parent rated BRIEF in a small sample of 1216 year olds with VI suggests that this may be a longstanding and continuous pattern across later childhood (Greenaway et al 2016) and further research is required to identify the specific constraints underlying this apparent behavioral vulnerability.

The current investigation was constrained in several ways which potentially limits the generalizability of the findings. First, the sample size was limited to eighteen cases due to the recruitment challenges of the very rare 'simple' congenital disorders of the peripheral visual system (Rahi, Cable, BCVISG, 2003); other studies on VI are often of similar size for similar reasons (Tadic, Pring, \& Dale, 2009; Absoud, Parr, Salt, \& Dale, 2011). Because of this small sample size, only large effects between group means could be detected and investigation of subtler group differences may have been underpowered (Button, Ioannidis, Mokrysz, Nosek, Flint, Robinson, \& Munafo, 2013). Further, in order to recruit a sufficient number of individuals, a range of congenital visual disorders were included that shared common functional symptoms. Despite this heterogeneity, overall similarity 
of the test scores across the VI sample suggests that common functional issues (VI and degree of severity of vision reduction) are of greater relevance than individual anatomical disorders of globe, retina or optic nerve (Sonksen and Dale 2002).

Second, a further limitation of the study is the absence of EF tests that have been designed for and validated on children with VI. However, the similar performance between the VI sample and the TS control group on most of the standard tasks implies that validity and reliability were unlikely to be seriously constrained. This also meant that some areas of EF such as set shifting, problem solving and design fluency could not be assessed due to lack of suitable tests; the current study can therefore not be viewed as a broadly comprehensive investigation of EF abilities in children with VI.

Particularly striking was the discrepancy of results between the neuropsychological tests and the behavioral questionnaire, which may reflect methodological issues. The questionnaire measure may tap different dimensions or constructs related to EF compared to standard assessment or lab-based measures (Eycke and Dewey, 2015; Toplak, Bucciarelli, \& Jain, 2008; Chan et al 2008, Toplak \& West, 2013). This is supported by similar discrepancies that have been reported in other clinical populations e.g. frontal lobe patients (Shallice and Burgess 1991, Chan et al., 2008). However, parent ratings may also be less accurate than direct standardized testing and might reflect unrealistic parental expectations of their child with VI's performance.

\section{Conclusion}

The present study is the first study, to the authors' knowledge, to report on EF abilities based on systematic neuropsychological assessments in a group of higher functioning children with congenital VI and to relate this to current precise levels of vision reduction. The study provides persuasive evidence that children with VI, including with severe to profound vision reduction, could succeed in auditory and verbal neuropsychological tests of working memory, attention and verbal fluency to the same level as matched controls with typical sight.

The results of the current investigation have potentially important implications for clinical and educational practice. The results of the parent behavioural questionnaire may indicate that even though a child may be doing relatively well at school on academic tasks, some of their behavioural EF abilities may not be developing as smoothly and any constraint in this area could impact on secondary school years where higher autonomy and independence is required. Further research would be useful in a larger sample of 1115 year olds to investigate whether children can apply their cognitive or behavioural EF abilities in the secondary school environment. In middle childhood, parents may be the first to be concerned about their child's difficulties at home, but educators or clinicians also need to be alerted to the child struggling in sustaining or dividing their attention in a 491 busy classroom, or taking initiative, or shifting between mental sets or tasks, or generat- 
492 ing new ideas to devise and follow goal-directed plans. Of further clinical concern, EF

493 difficulties in older children with VI predict greater behaviour problems and socio-

494 emotional difficulties (Heyl and Hintermair, 2015). In these circumstances, a specialised

495 clinical neuropsychological assessment could be valuable in identifying needs and

496 providing guidance or intervention for supporting EF abilities. Further research would

497 be beneficial for developing and evaluating interventions to assist the more vulnerable

498 school aged children with VI and weaker EF abilities. Greater severity of VI is a particular

499 risk factor, but even in mild-moderate VI some children struggle in this area.

This study limited itself to higher functioning children with VI and there are many children with VI who also have additional neurological impairment (Rahi and Cable 2003); it is predicted that they will struggle to a greater extent with EF related abilities (Heyl and Hintermair, 2015). Autism related difficulties are present in a significant pro-

\section{Acknowledgements}

This research was funded by Great Ormond Street Hospital Children's Charity, UCL Impact, UCL Grand Challenges, the Royal National Institute of Blind People, and supported by the National Institute for Health Research Biomedical Research Centre at Great Ormond Street Hospital for Children NHS Foundation Trust and University College Lon518 don. 


\section{References}

Absoud, M., Parr, J. R., Salt, A., \& Dale, N. (2011). Developing a schedule to identify social communication difficulties and autism spectrum disorder in young children with visual impairment. Developmental Medicine \& Child Neurology, 53(3), 285-288. doi:10.1111/j.1469-8749.2010.03846.x

Alimovic, S. (2013). Emotional and behavioural problems in children with VI, intellectual and multiple disabilities. Journal of Intellectual Disability Research, 57(2), 153-160. http://doi.org/10.1111/j.1365-2788.2012.01562.x

Amedi, A., Raz, N., Pianka, P., Malach, R., \& Zohary, E. (2003). Early "visual" cortex activation correlates with superior verbal memory performance in the blind. Nature Neuroscience, 6(7), 758-766. http://doi.org/10.1038/nn1072

Anderson, P. (2002). Assessment and development of EF (EF) during childhood. Child Neuropsychology, 8(2), 71-82. http://doi.org/10.1076/chin.8.2.71.8724

Bertone, A., Bettinelli, L., \& Faubert, J. (2007). The impact of blurred vision on cognitive assessment. Journal of Clinical and Experimental Neuropsychology, 29(5), 467-476. http://doi.org/10.1080/13803390600770793

Button, K. S., Ioannidis, J. P. A., Mokrysz, C., Nosek, B. A., Flint, J., Robinson, E. S. J., \& Munafò, M. R. (2013). Power failure: why small sample size undermines the reliability of neuroscience. Nature Reviews Neuroscience, 14(5), 365-376. http://doi.org/10.1038/nrn3475

Cavezian, C., Vilayphonh, M., Vasseur, V., Caputo, G., Laloum, L., \& Chokron, S. (2013). Ophthalmic disorder may affect visuo-attentional performance in childhood. Child Neuropsychology : a Journal on Normal and Abnormal Development in Childhood and Adolescence, 19(3), 292-312. http://doi.org/10.1080/09297049.2012.670214

Chronbach, L.J., Meehl, P.E. (1955). Construct validity in psychological tests. Psychol. Bull. 52, 281-302

Cohen, M. (1997). Children's Memory Scale (CMS). Oxford, UK: Pearson Assessment, UK. Retrieved from http://www.pearsonclinical.co.uk/Psychology/ChildCognitionNeuropsychologyand Language/ChildMemory/ChildrensMemoryScale(CMS)/ChildrensMemoryScale(CMS).a $\operatorname{spx}$

Colombo, J. (2001). The Development of Visual Attention in Infancy. Annual Review of Psychology,52(1), 337-367. doi:10.1146/annurev.psych.52.1.337

Dale, N., \& Sonksen, P. (2002). Developmental outcome, including setback, in young children with severe VI., 44(9), 613-622.

de Haan, M. (2014). Attention and Executive Control. In D. Mareschal, B. Butterworth, \& A. Tolmie, Educational Neuroscience. Chichester, UK.

Decarlo, D. K., Bowman, E., Monroe, C., Kline, R., McGwin, G., \& Owsley, C. (2014). Prevalence of attention-deficit/hyperactivity disorder among children with vision impairment. Journal of AAPOS : the Official Publication of the American Association for Pediatric Ophthalmology and Strabismus / American Association for Pediatric Ophthalmology and Strabismus, 18(1), 10-14. http://doi.org/10.1016/j.jaapos.2013.10.013

Dekker, R. (1993). Visually impaired children and haptic intelligence test scores: intelligence test for visually impaired children (ITVIC)., 35(6), 478-489.

Delis, D. C., Lansing, A., Houston, W. S., Wetter, S., Han, S. D., Jacobson, M., et al. (2007). Creativity lost: The importance of testing higher-level EFs in school-age children and adolescents. Journal of Psychoeducational Assessment, 25(1), 29-40.

Delis, D., Kramer, J. H., \& Kaplan, E. (2001). The Delis-Kaplan EF System.

Diamond, A. (2013). EFs. Annual Review of Psychology, 64(1), 135-168. http://doi.org/10.1146/annurev-psych-113011-143750 
Eycke, Ten, K. D., \& Dewey, D. (2015). Parent-report and performance-based measures of executive function assess different constructs. Child Neuropsychology, 1-18. http://doi.org/10.1080/09297049.2015.1065961

Garcia-Filion, P., \& Borchert, M. (2013). Optic nerve hypoplasia syndrome: a review of the epidemiology and clinical associations. Current Treatment Options in Neurology, 15(1), 78-89. http://doi.org/10.1007/s11940-012-0209-2

Gioia, G. A., Isquith, P. K., \& Kenworthy, L. (2000). Behavior Rating of EF.

Gioia, G. A., Isquith, P. K., Kenworthy, L., \& Barton, R. M. (2002a). Profiles of everyday EF in acquired and developmental disorders, $8(2), 121-137$.

Gioia, G. A., Isquith, P. K., Retzlaff, P. D., \& Espy, K. A. (2002b). Confirmatory factor analysis of the Behavior Rating Inventory of EF (BRIEF) in a clinical sample. Child Neuropsychology, 8(4), 249-257. http://doi.org/10.1076/chin.8.4.249.13513

Gomes, H., Molholm, S., Christodoulou, C., Ritter, W., \& Cowan, N. (2000). The development of auditory attention in children. Frontiers in Bioscience : a Journal and Virtual Library, 5, D108-20.

Greenaway, R., Pring, L., Schepers, A., Isaacs, D. P., \& Dale, N. J. (2016). Neuropsychological Presentation and Adaptive Skills in High-Functioning Adolescents with Visual Impairment: A Preliminary Investigation. Applied Neuropsychology: Child. http://doi.org/10.1080/21622965.2015.1129608

Greenhouse, S. W., \& Geisser, S. (1959). On methods in the analysis of profile data. Psychometrika, 24(2), 95-112. http://doi.org/10.1007/BF02289823

Harrell, F. E. (2001). Regression Modeling Strategies. Springer Science \& Business Media.

Hecke, V. A., Mundy, PC, \& Acra, CF. (2007). Infant joint attention, temperament, and social competence in preschool children. Child Development, 78 (1), doi:10.1111/j.1467-8624.2007.00985.x

Henry, L., Messer, D., \& Nash, G. (2015). Executive functioning and verbal fluency in children with language difficulties. Learning and Instruction, 39, 137-147. doi:10.1016/j.learninstruc.2015.06.00

Heyl, V. \& Hintermair, M. (2015). Executive functions and behavior problems in students with visual impairments at regular and special schools. Journal of Visual Impairment and Blindness, 109, 251-263.

Isquith, P. K., Roth, R. M., \& Gioia, G. (2013). Contribution of rating scales to the assessment of EFs. Applied Neuropsychology. Child, 2(2), 125-132. http://doi.org/10.1080/21622965.2013.748389

Johnson, M. H., \& de Haan, M. (2011). Developmental Cognitive Neuroscience (3rd ed.). Wiley-Blackwell.

Jurado, M. B., \& Rosselli, M. (2007). The Elusive Nature of Executive Functions: A Review of our Current Understanding. Neuropsychology Review, 17(3), 213-233. http://doi.org/10.1007/s11065-007-9040-z

Kleinhans, N., Akshoomoff, N., \& Delis, D. C. (2005). EFs in autism and Asperger's disorder: flexibility, fluency, and inhibition. Developmental Neuropsychology, 27(3), 379-401. http://doi.org/10.1207/s15326942dn2703_5

Kramer, J. H., Quitania, L., Dean, D., Neuhaus, J., Rosen, H. J., Halabi, C., et al. (2007). Magnetic resonance imaging correlates of set shifting. Journal of the International Neuropsychological Society, 13(3), 386-392. http://doi.org/10.1017/S1355617707070567

Kravitz, D. J., Saleem, K. S., Baker, C. I., \& Mishkin, M. (2011). A new neural framework for visuospatial processing, 12, 217-230. http://doi.org/10.1038/nrn3008

Liu, Y., Yu, C., Liang, M., Li, J.,Tian, L., Zhou, Y., Qin, W., Li, K., Jiang, T. (2007). Whole brain functional connectivity in the early blind. Brain 130, 2085-2096.

Lopez, B. R., Lincoln, A. J., Ozonoff, S., \& Lai, Z. (2005). Examining the relationship between EFs and restricted, repetitive symptoms of Autistic Disorder. Journal of Autism and Developmental Disorders, 35(4), 445-460. 
http://doi.org/10.1007/s10803-005-5035-x

Manly, T., Nimmo-Smith, I., Turner, A., Watson, P., \& Robertson, I. H. (2001). The differential assessment of children's attention: the Test of Everyday Attention for Children (TEA-Ch), normative sample and ADHD performance. Journal of Child Psychology and Psychiatry, and Allied Disciplines, 42(8), 1065-1081. http://doi.org/10.1111/1469-7610.00806

Matute, E., Rosselli, M., Ardila, A., \& Morales, G. (2004). Verbal and nonverbal fluency in Spanish-speaking children. Developmental Neuropsychology, 26(2), 647-660. http://doi.org/10.1207/s15326942dn2602_7

Mauchly, J. W. (1940). Significance Test for Sphericity of a Normal n-Variate Distribution, 11(2), 204-209. http://doi.org/10.1214/aoms/1177731915

McDermott, J. M., Westerlund, A., Zeanah, C. H., \& Fox, N. A. (2012). Early adversity and neural correlates of EF: implications for academic adjustment., 2 Suppl 1, S59-66. http://doi.org/10.1016/j.dcn.2011.09.008

McDonald, C. R., Delis, D. C., Norman, M. A., Wetter, S. R., Tecoma, E. S., \& Iragui, V. J. (2005). Response inhibition and set shifting in patients with frontal lobe epilepsy or temporal lobe epilepsy. Epilepsy and Behavior, 7(3), 438-446. http://doi.org/10.1016/j.yebeh.2005.05.005

Miyake, A., Friedman, N. P., Emerson, M. J., Witzki, A. H., Howerter, A., \& Wager, T. D. (2000). The unity and diversity of EFs and their contributions to complex "Frontal Lobe" tasks: a latent variable analysis. Cognitive Psychology, 41(1), 49-100. http://doi.org/10.1006/cogp.1999.0734

Mukkades, N. M., Kilincaslan, A., Kucukaltun-Yildirim, E., Sevketoglu, T., \& Tuncer, S. (2007). Autism in visually impaired individuals. Psychiatry and Clinical Neurosciences, 61(1), 39-44. http://doi.org/10.1111/j.1440-1819.2007.01608.x

Mundy, P, Block, J, Delgado, C, \& Pomares, Y. (2007). Individual differences and the development of joint attention in infancy. Child Development 78(3), http://doi.org/10.1111/j.1467-8624.2007.01042.x

Nakagawa, A., \& Sukigara, M. (2013). Individual differences in disengagement of fixation and temperament: Longitudinal research on toddlers.Infant Behavior and Development, 36(4), 728-735. doi:10.1016/j.infbeh.2013.08.001

Noppeney, U. (2007). The effects of visual deprivation on functional and structural organization of the human brain. Neuroscience \& Biobehavioral Reviews 31, 116911 ic8

Ozonoff, S., Pennington, B. F., \& Rogers, S. J. (1991). Executive function deficits in highfunctioning autistic individuals: relationship to theory of mind. Journal of Child Psychology and Psychiatry, and Allied Disciplines, 32(7), 1081-1105.

Perez-Pereira, M., Conti-Ramsden, G. (1999). Language development and social interaction in children. Psychology Press. East Sussex, UK

Papageorgiou, K. A., Smith, T. J., Wu, R., Johnson, M. H., Kirkham, N. Z., \& Ronald, A. (2014). Individual differences in infant fixation duration relate to attention and behavioral control in childhood.Psychological science, 25(7), 1371-9. doi:10.1177/0956797614531295

Parr, J. R., Dale, N. J., Shaffer, L. M., \& Salt, A. T. (2010). Social communication difficulties and autism spectrum disorder in young children with optic nerve hypoplasia and/or septo-optic dysplasia., 52(10), 917-921. http://doi.org/10.1111/j.14698749.2010.03664.x

Ptak, R. (2012). The Frontoparietal Attention Network of the Human Brain: Action, Saliency, and a Priority Map of the Environment. The Neuroscientist, 18(5), 502-515. http://doi.org/10.1177/1073858411409051

Rahi, J. S., Cable, N., BCVISG. (2003). Severe VI and blindness in children in the UK. The Lancet, 362(9393), 1359-1365. http://doi.org/10.1016/S0140-6736(03)14631-4

Raz, N., Striem, E., Pundak, G., Orlov, T., \& Zohary, E. (2007). Superior serial memory in 
the blind: a case of cognitive compensatory adjustment., 17(13), 1129-1133. http://doi.org/10.1016/j.cub.2007.05.060

Richards, J. E., Reynolds, G. D., \& Courage, M. L. (2010). The Neural Bases of Infant Attention. Current Directions in Psychological Science, 19(1), 41-46. http://doi.org/10.1177/0963721409360003

Shu, N., Li, J., Li, K., Yu, C., Jiang, T. (2009). Abnormal diffusion of cerebral white matter in early blindness. Human Brain Mapping 30, 220-227.

Smith, L, \& Ulvund, SE. (2003). The role of joint attention in later development among preterm children: Linkages between early and middle childhood. Social Development, 12(2), http://doi.org/10.1111/1467-9507.00230

Sonksen, P. M., \& Dale, N. (2002). VI in infancy: impact on neurodevelopmental and neurobiological processes. Developmental Medicine \& Child Neurology, 44(11), 782791. http://doi.org/j.1469-8749.2002.tb00287.x

Sonksen, P. M., Petrie, A., \& Drew, K. J. (1991). Sonksen Near Detection Scale - distinguishing between severe and profound levels of VI, 33(4), 320-335.

Sonksen, P. M., Wade, A. M., Proffitt, R., Heavens, S., \& Salt, A. T. (2008). The Sonksen $\log$ MAR test of visual acuity: II. Age norms from 2 years 9 months to 8 years. Journal of AAPOS : the Official Publication of the American Association for Pediatric Ophthalmology and Strabismus / American Association for Pediatric Ophthalmology and Strabismus, 12(1), 18-22. http://doi.org/10.1016/j.jaapos.2007.04.019

Stevens, C., Lauinger, B., \& Neville, H. (2009). Differences in the neural mechanisms of selective attention in children from different socioeconomic backgrounds: an eventrelated brain potential study. Developmental Science, 12(4), 634-646. http://doi.org/10.1111/j.1467-7687.2009.00807.x

Swanson, H. L., \& Luxenberg, D. (2009). Short-term memory and working memory in children with blindness: support for a domain general or domain specific system?, 15(3), 280-294. http://doi.org/10.1080/09297040802524206

Tadic, V. (2009). Development of social functioning in children with congenital visual impairment (Ph.D. thesis, Goldsmiths College, University of London, London, U.K.). Retrieved from http://ethos.bl.uk/OrderDetails.do?uin=uk.bl.ethos.514388

Tadic, V., Pring, L., \& Dale, N. (2009). Attentional processes in young children with congenital VI. The British Journal of Developmental Psychology, 27(Pt 2), 311-330. http://doi.org/10.1348/026151008X310210

The R Development Core Team. (2008). R: A language and environment for statistical computing. Vienna, Austria: R Foundation for Statistical Computing. Retrieved from http://www.R-project.org.

Tillman, M. H. (1973). Intelligence scales for the blind: A review with implications for research, 11(1), 80-87. http://doi.org/10.1016/0022-4405(73)90014-9

Tillman, M. H., \& Bashaw, W. L. (1968). Multivariate analysis of the WISC scales for blind and sighted children, 23(2), 523-526.

Toplak, M. E., \& West, R. F. (2013). Practitioner Review: Do performance-based measures and ratings of executive function assess the same construct? Journal of Child Psychology and Psychiatry, http://doi.org/10.1111/jcpp.12001

Toplak, M. E., Bucciarelli, S. M., \& Jain, U. (2008). Executive Functions: PerformanceBased Measures and the Behavior Rating Inventory of Executive Function (BRIEF) in Adolescents with Attention Deficit/Hyperactivity Disorder (ADHD) Child Neuropsychology, 15(1), 54-72. http://doi.org/10.1080/09297040802070929

Warren, D. H. (1994). Blindness and Children: An Individual Differences Approach. Cambridge University Press, New York

Wechsler, D. (2004). The Wechsler Intelligence Scale for Children - fourth edition.

Welch, B. L. (1947). The generalisation of 'student's' problems when several different population variances are involved. Biometrika, 34(1-2), 28-35. http://doi.org/10.1093/biomet/34.1-2.28 
733 Wickham, H. (2009). ggplot2: elegant graphics for data analysis. New York, NY: Springer. Retrieved from http://had.co.nz/ggplot2/book the executive function theory of attention-deficit/hyperactivity disorder: a metaanalytic review. Biological Psychiatry, 57(11), 1336-1346. http://doi.org/10.1016/j.biopsych.2005.02.006

Witkin, H. A., Birnbaum, J., Lomonaco, S., Lehr, S., \& Herman, J. L. (1968). Cognitive patterning in congenitally totally blind children., 39(3), 766-786.

Xu, F., Han, Y., Sabbagh, M., Wang, T., Ren, X., \& Li, C. (2013). Developmental differences in the structure of executive function in middle childhood and adolescence. PLoS ONE, 8(10), e77770. doi:10.1371/journal.pone.007777 
$748 \quad$ Figure captions

\section{Figure 1:}

750 a) Results of the sustained and divided auditory attention task

751 The distribution of standardized tests scores in the sustained and divided auditory atten752 tion condition are shown for the VI group (black) and the control group (grey). The solid 753 grey line indicates the mean of the normative sample. The dashed lines show one stand754 ard deviations variance of the mean of the normative sample (Robertson, Ward, Ridge755 way, \& Nimmo-Smith, 1994). There were no significant differences between groups in 756 either condition.

757 b) Results of the semantic, phonemic, and switching verbal fluency assessment

758 The distribution of standardized scores is shown for the VI (black) and control group 759 (grey) in the phonemic, semantic, and switching conditions. Number of response and 760 switching accuracy are shown separately for the switching condition (Delis et al., 2001). 761 The solid grey line indicates the mean of the normative sample and the dashed lines 762 show one standard deviation variance from the norm mean.

\section{3 c) Results of the everyday executive ability parent questionnaire}

764 The mean score and standard error on each scale is shown for children in the VI (black) 765 and control group (grey). The solid grey line indicates the mean scores of the normative 766 sample, the dashed grey lines show one standard deviation of variance from the mean. 767 Scales on the left of the vertical black line made up the Behavioral Regulation Index, 768 while scales to the right were summarized in the Metacognitive Index (Gioia et al., 2000). 769 There were significant differences on all scales, except for Organization of Materials. 
771 Table captions

772 Table 1: Characteristics of participants in the VI group

773 Demographic information and results of verbal ability and visual acuity assessments are

774 listed. Abbreviations: MVI: mild/moderate VI (degraded visual acuity); SVI: severe VI

775 (basic form vision); PVI: profound VI (light perception at best); WISC: Wechsler Intelli-

776 gence Scale for Children 4th edition;

777

778 Table 2: Characteristics of the typically-sighted (TS) control group

779 Table 3: Descriptive statistics of mean scores and standard errors of the

780 mean (SE) across executive function measures

781

782

783 
a

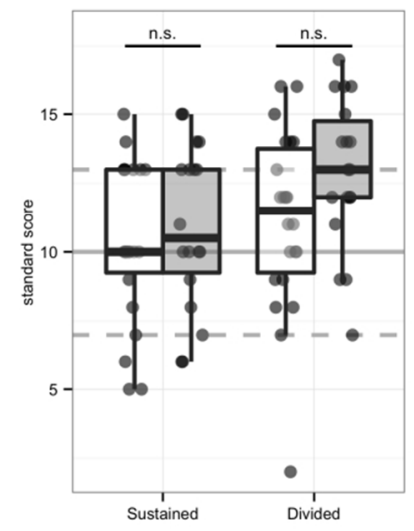

b

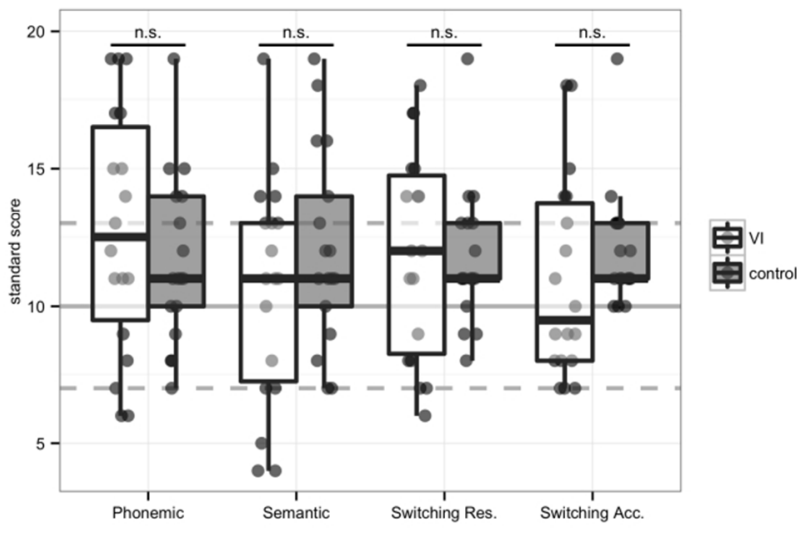

C

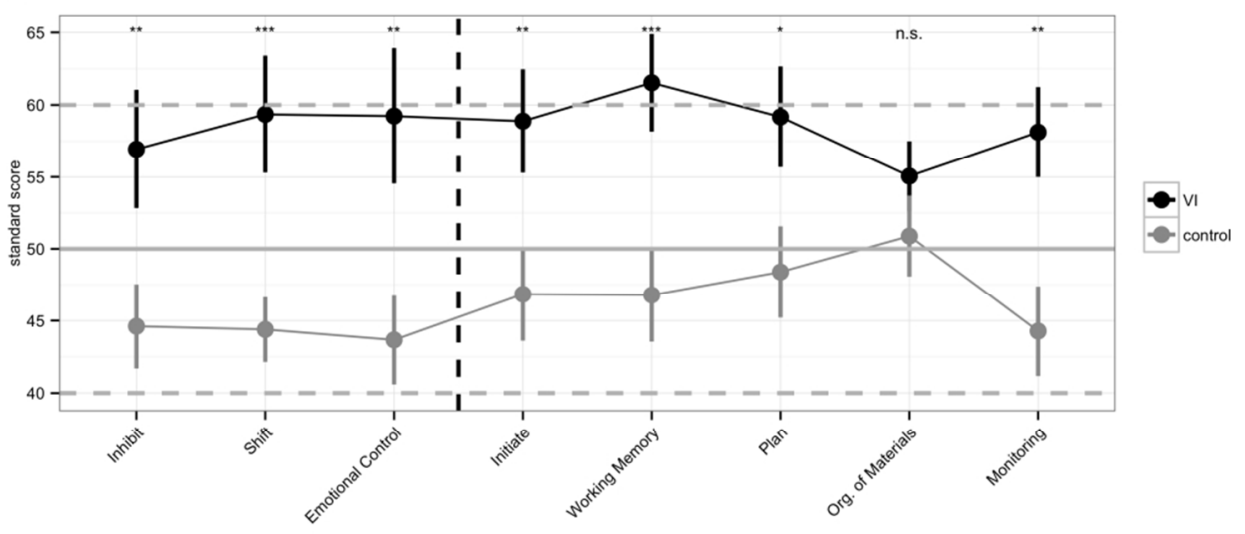

a) Results of the sustained and divided auditory attention task

The distribution of standardized tests scores in the sustained and divided auditory attention condition are shown for the VI group (black) and the control group (grey). The solid grey line indicates the mean of the normative sample. The dashed lines show one standard deviations variance of the mean of the normative sample (Robertson, Ward, Ridgeway, \& Nimmo-Smith, 1994). There were no significant differences between groups in either condition.

b) Results of the semantic, phonemic, and switching verbal fluency assessment

The distribution of standardized scores is shown for the VI (black) and control group (grey) in the phonemic, semantic, and switching conditions. Number of response and switching accuracy are shown separately for the switching condition (Delis et al., 2001). The solid grey line indicates the mean of the normative sample and the dashed lines show one standard deviation variance from the norm mean.

c) Results of the everyday executive ability parent questionnaire

The mean score and standard error on each scale is shown for children in the VI (black) and control group (grey). The solid grey line indicates the mean scores of the normative sample, the dashed grey lines show one standard deviation of variance from the mean. Scales on the left of the vertical black line made up the Behavioral Regulation Index, while scales to the right were summarized in the Metacognitive Index (Gioia et al., 2000). There were significant differences on all scales, except for Organization of Materials. 
Table 1: VI group characteristics

\begin{tabular}{|c|c|c|c|c|c|c|c|}
\hline ID & Gender & Age $[y]$ & VerbComp & $\log \mathrm{Mar}$ & Near Detection & Vision Group & Visual Disorder \\
\hline MVI 1 & female & 9.19 & 114 & 0.1 & - & MVI & congenital nystagmus \\
\hline MVI 2 & female & 13.32 & 95 & 0.4 & - & MVI & Ocular fibrosis \\
\hline MVI 3 & female & 11.91 & 104 & 0.5 & - & MVI & bilateral optic nerve hypoplasia \\
\hline MVI 4 & male & 12.34 & - & 0.54 & - & MVI & rod-cone dystrophy \\
\hline MVI 5 & female & 8.27 & 104 & 0.6 & - & MVI & oculocutaneous albinisim \\
\hline MVI 6 & male & 12.06 & 104 & 0.6 & - & MVI & congenital nystagmus \\
\hline MVI 7 & male & 10.64 & 116 & 0.6 & - & MVI & congenital nystagmus \\
\hline MVI 8 & male & 9.82 & 93 & 0.7 & - & MVI & ocular albinism, congenital nystagmus \\
\hline MVI 9 & female & 12.26 & 96 & - & $\begin{array}{l}\text { left: } 0.23 \text {, } \\
\text { right: light perception }\end{array}$ & $\begin{array}{l}\text { MVI, } \\
\text { PVI }\end{array}$ & unilateral optic nerve hypoplasia \\
\hline SVI 1 & female & 10.98 & 87 & 0.9 & - & SVI & hereditary progressive cone dystrophy \\
\hline SVI 2 & male & 11.69 & 148 & 0.9 & - & SVI & oculocutaneous albinisim \\
\hline SVI 3 & female & 10.98 & 78 & 1.1 & - & SVI & FEVR, LRP5 mutation \\
\hline SVI 4 & male & 9.57 & 119 & 1.2 & - & SVI & Leber's congenital amaurosis \\
\hline SVI 5 & male & 9.01 & - & 1.225 & - & SVI & ocular albinism, nystagmus \\
\hline SVI 6 & male & 9.91 & 96 & 1.225 & - & SVI & Norrie's disease \\
\hline SVI 7 & female & 11.04 & 75 & - & $1.5 \mathrm{~cm}$ sweet from $20 \mathrm{~cm}$ & SVI & Leber's congenital amaurosis \\
\hline \multirow[t]{2}{*}{ PVI 1} & male & 10.36 & 134 & - & light perception only & PVI & Leber's congenital amaurosis \\
\hline & $\begin{array}{l}9 \text { female } \\
9 \text { male }\end{array}$ & $\begin{array}{r}\text { mean }=10.73 \\
\mathrm{SE}=0.31\end{array}$ & $\begin{array}{r}\text { mean }=103.63 \\
\mathrm{SE}=4.41\end{array}$ & & & & 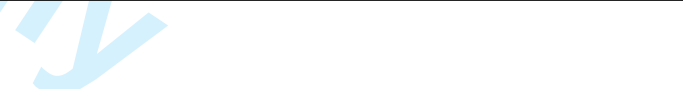 \\
\hline
\end{tabular}

Abbreviations: MVI: mild visual impairment, SVI: severe visual impairment, PVI: profound visual impairment, VerbComp: WISC-IV

Verbal Comprehension age-normed score, FEVR: familial exudative vitreoretinopathy 


\section{Table 2: Control group characteristics}

\begin{tabular}{lrrrr}
\hline ID & Gender & Age [y] & VerbComp & logMAR \\
\hline C 1 & female & 8.56 & 98 & -0.3 \\
C 2 & female & 8.73 & 110 & 0.1 \\
C 3 & male & 8.90 & 116 & -0.3 \\
C 4 & male & 9.08 & 102 & 0.1 \\
C 5 & female & 9.12 & 98 & -0.1 \\
C 6 & male & 9.34 & 108 & -0.2 \\
C 7 & male & 10.07 & 96 & 0.1 \\
C 8 & male & 10.16 & 134 & 0.0 \\
C 9 & male & 10.37 & 106 & 0.0 \\
C 10 & male & 10.74 & 102 & -0.2 \\
C 11 & female & 10.78 & 134 & 0.1 \\
C 12 & female & 10.82 & 116 & -0.2 \\
C 13 & female & 10.89 & 83 & 0.0 \\
C 14 & female & 11.09 & 130 & -0.3 \\
C 15 & female & 11.78 & 144 & 0.1 \\
C 16 & male & 12.70 & 106 & -0.2 \\
C 17 & male & 12.77 & 130 & -0.2 \\
C 18 & male & 12.92 & 124 & -0.3 \\
\hline
\end{tabular}

8 female mean $=10.49 \quad$ mean $=113.17$

10 male $\quad \mathrm{SE}=0.32 \quad \mathrm{SE}=3.87$ 
Table 3: Results of executive function assessment

\begin{tabular}{|c|c|c|c|c|c|c|c|}
\hline & & \multicolumn{2}{|c|}{$\mathrm{S} / \mathrm{PVI}$} & \multicolumn{2}{|c|}{ MVI } & \multicolumn{2}{|c|}{ control } \\
\hline & & mean & SE & mean & $\mathrm{SE}$ & mean & $\mathrm{SE}$ \\
\hline \multirow[t]{3}{*}{ WM } & Total & 99.1 & 12.59 & 101.25 & 4.08 & 100.50 & 2.41 \\
\hline & Digit Span & 10.44 & 1.21 & 8.75 & 0.70 & 9.22 & 0.66 \\
\hline & L-N Seq & 9.56 & 1.59 & 10.12 & 0.97 & 11.17 & 0.32 \\
\hline \multirow[t]{2}{*}{ Aud Att } & Sustained & 10.11 & 1.02 & 10.38 & 0.84 & 10.94 & 0.70 \\
\hline & Divided & 10.78 & 1.39 & 11.00 & 1.10 & 12.89 & 0.64 \\
\hline \multirow[t]{4}{*}{ Verb Fl } & Letter & 12.75 & 1.95 & 13.00 & 1.30 & 12.63 & 0.99 \\
\hline & Category & 9.25 & 1.86 & 11.12 & 0.93 & 12.06 & 0.87 \\
\hline & Swtch Resp & 11.12 & 1.52 & 12.50 & 1.32 & 11.76 & 0.62 \\
\hline & Swtch Acc & 10.50 & 1.50 & 12.00 & 1.16 & 12.06 & 0.52 \\
\hline \multirow[t]{3}{*}{ BRIEF } & GEC & 62.86 & 5.43 & 58.12 & 7.33 & 45.11 & 3.25 \\
\hline & BRI & 59.14 & 6.51 & 58.88 & 6.96 & 42.83 & 4.10 \\
\hline & MI & 62.86 & 4.68 & 57.75 & 6.85 & 43.50 & 1.93 \\
\hline
\end{tabular}

Abbreviations: Aud Att: Auditory Attention; BRI: Behavioral Regulation Index; BRIEF: Behavior Rating Inventory of Executive Function; EF: Executive Function; GEC: Global Executive Composite; L-N Seq: Letter-Number Sequence; MI: Metacognitive Index; MVI: mild-to-moderate visual impairment; S/PI: severe-to-profound visual impairment; SE: standard error of the mean; Swtch Acc: Switching Accuracy; Swtch Resp: Switching Responses; Verb Fl: Verbal Fluency; VI: visual impairment, WM: Working Memory 